\title{
Reinforcement in melt-processed polymer-graphene composites at extremely low graphene loading level
}

Oana M. Istrate ${ }^{a, b}$, Keith R. Paton ${ }^{a, c}$, Umar Khan $^{a, b}$, Arlene O'Neill ${ }^{a, b}$, Alan P. Bell ${ }^{a, d}$ and Jonathan N. Coleman ${ }^{a, b, *}$

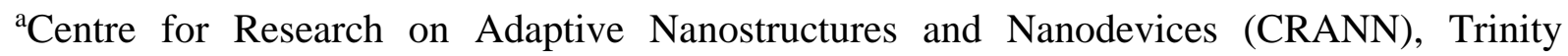
College Dublin, Dublin 2, Ireland;

${ }^{\mathrm{b} S c h o o l ~ o f ~ P h y s i c s, ~ T r i n i t y ~ C o l l e g e ~ D u b l i n, ~ D u b l i n ~ 2, ~ I r e l a n d ; ~}$

${ }^{\mathrm{c}}$ Thomas Swan \& Co. Ltd., Rotary Way, Consett, County Durham, DH8 7ND, United Kingdom;

${ }^{\mathrm{d} A d v a n c e d ~ M i c r o s c o p y ~ L a b o r a t o r y}$ (AML), Trinity Enterprise Centre Unit 27/29, Trinity College Dublin, Dublin 2, Ireland

*Corresponding author:

J. N. Coleman

Fax: +353(0)1671 1759

E-mail: $\underline{\text { colemaj@tcd.ie }}$ 


\begin{abstract}
We have prepared polymer nanocomposites reinforced with exfoliated graphene layers solely via melt blending. For this study polyethylene terephthalate (PET) was chosen as the polymer matrix due to its myriad of current and potential applications. PET and PET/graphene nanocomposites were melt compounded on an internal mixer and the resulting materials were compression molded into films. Transmission electron microscopy and scanning electron microscopy revealed that the graphene flakes were randomly orientated and well dispersed inside the polymer matrix. The PET/graphene nanocomposites were found to be characterized by superior mechanical properties as opposed to the neat PET. Thus, at a nanofiller load as low as $0.07 \mathrm{wt} \%$, the novel materials presented an increase in the elastic modulus higher than $10 \%$ and an enhancement in the tensile strength of more than $40 \%$ compared to pristine PET. The improvements in the tensile strength were directly correlated to changes in elongation at break and indirectly correlated to the fracture initiation area. The enhancements observed in the mechanical properties of polymer/graphene nanocomposites achieved at low exfoliated graphene loadings and manufactured exclusively via melt mixing may open the door to industrial manufacturing of economical novel materials with superior stiffness, strength and ductility.
\end{abstract}




\section{Introduction}

Among its other superlative properties [1-3], graphene is the strongest material known to man [4]. As a result, it has long been known that graphene has the potential to be a superlative filler in reinforced composites. To this end, much work has studied the effect of graphene on the mechanical properties of polymer matrices [5]. Although, such research has progressed in many directions, two of the most common aims are either to produce high-performance composites such as high-strength, polymer-graphene fibers or to achieve modest levels of reinforcement but at very low graphene loading levels. The latter goal is important for a number of applications such as in hierarchical composites, where the main reinforcement is due to relatively large fillers such as carbon fibers. However, achieving relatively small increases in stiffness and strength of the matrix by adding a nano-filler can lead to non-trivial increases in the ultimate composite properties. This should be achieved at very low loading levels, both for reasons of cost and to avoid significantly altering other matrix properties.

Typically, polymer/graphene nanocomposites have been manufactured using covalently or non-covalently modified graphene via in situ polymerization, solution blending or melt compounding or by a combination of these manufacturing techniques. This work has been extensively reported in articles and in literature reviews [5-9]. From an industrial point of view, melt blending is the only practical approach as it does not make use of high quantities of solvents nor does it require the development of a dedicated line in a polymer manufacturing facility. However, to date, relatively few papers have been published on polymer/graphene nanocomposites manufactured solely via melt processing, i.e. without a solution-processing step to the composite formation procedure [10-14]. None of these papers have shown significant degrees of reinforcement at very low volume fraction $(<0.1 \%)$. In this paper, we report the fabrication of polymer/graphene nanocomposites via melt compounding, using pristine graphene layers at weight fractions lower than $0.1 \mathrm{wt} \%$. The polymer matrix used in this study was polyethylene terephthalate (PET), a choice which was made for a number of reasons. Firstly, previous work has shown that graphene can be used to effectively reinforce PET [15]. In addition, PET is widely used in applications where mechanical properties are relevant and so reinforcement of PET could prove highly useful. Also, the structure of PET and previous research on graphene and PET [15] leads us to expect reasonable interactions between the graphene and the polymer matrix, for example between the graphene surface and the aromatic groups present on the PET chains [16]. We find that by dispersing extremely low volume fractions of exfoliated graphene into the polyethylene terephthalate matrix via melt 
blending, materials with significantly improved mechanical properties are formed. This approach is simple and compatible with industrial processes and paves the way to using exfoliated graphene layers at an industrial scale. Interestingly, the reinforcement mechanism is distinct from that normally found in nanocomposites and is associated with modifications to the fracture process due to the presence of graphene.

\section{Methods}

Graphite was purchased from Sigma Aldrich Ltd. (product code 332461). The exfoliated graphene layers have been manufactured, in house, using liquid exfoliation in the solvent $\mathrm{N}$ methyl-pyrrolidone (NMP) [17-19]. Basically, powdered graphite was shear mixed in the solvent NMP at an initial concentration of $100 \mathrm{~g} \cdot \mathrm{L}^{-1}$. The shear mixing was performed on a Silverson Mixer using a $32 \mathrm{~mm}$ rotor for 90 minutes at a speed of $6000 \mathrm{rpm}$ and a batch volume of $2.5 \mathrm{~L}$ according to a procedure previously reported in literature [19]. The solvent was removed by filtering the shear mixed dispersion through lab paper using a Buchner funnel. The resulting powder was dried at $60^{\circ} \mathrm{C}$ overnight in a vacuum oven.

Polyethylene terephthalate was purchased from Goodfellow Cambridge Ltd. (product code ES306310). PET and PET/graphene nanocomposites with a load varying from $0.01 \mathrm{wt} \%$ to $0.1 \mathrm{wt} \%$ were prepared by melt compounding, on a Brabender melt mixer, at a temperature of $260^{\circ} \mathrm{C}$. The melt mixing was performed by adding approximately half of the polymer quantity to the mixing bowl. Once the torque started to increase finely grinded graphene powder was added to the mixing bowl. When the polymer melted and the torque started to diminish, the remaining polymer was gradually added to the mixer. For all the materials the melt compounding was performed at an initial screw speed of $50 \mathrm{rpm}$ for 4 minutes, then the screw speed was increased to $80 \mathrm{rpm}$ within 1 minute and the melt mixing was conducted at this speed for another 5 minutes.

The materials obtained via melt compounding were compression molded at $280^{\circ} \mathrm{C}$ on an electrically heated hydraulic press. The samples were then cut into stripes with the following dimensions $50 \mathrm{~mm} \times 10 \mathrm{~mm} \times 0.5 \mathrm{~mm}(\mathrm{~L} \times \mathrm{w} \times \mathrm{t})$. Mechanical characterization was conducted on a Zwick Tensile Tester using a load cell of $2.5 \mathrm{kN}$, a gauge length of $20 \mathrm{~mm}$ and a speed of $5 \mathrm{~mm} \cdot \mathrm{min}^{-1}$. For all materials, 5 strips were mechanically tested.

Transmission electron microscopy (TEM) was performed on a TECNAI G2 20 Twin electron microscope in bright field mode at $200 \mathrm{kV}$. The sections were prepared using a Leica EM UC6 ultramicrotome cutter with a cryo-chamber (EM FC6). The cutting was performed at 
a temperature of $-40^{\circ} \mathrm{C}$ and a speed of $0.40 \mathrm{~mm} \cdot \mathrm{s}^{-1}$. The thin sections were placed on 200 mesh copper grids.

Scanning electron microscopy (SEM) was performed on tensile fractured surfaces using a Zeiss Ultra or a Zeiss Supra scanning electron microscope at a voltage of $5.0 \mathrm{kV}$ or $15.0 \mathrm{kV}$, respectively. Prior to being analyzed the samples were mounted on stubs and their surfaces were platinum coated.

Helium ion microscopy (HeIM) was carried on a Zeiss Orion Plus - helium ion microscope. The tensile fractured surfaces were mounted on stubs. No coating was necessary in this case.

\section{Results and discussion}

As described in the experimental section, the graphene powder was produced by liquid exfoliation [17-19]. This process is ideal in this case as it is known to produce graphene which is largely defect free [17, 20]. TEM analysis (Figure 1A-B) showed the graphene flakes produced by this method to be of good quality with mean lateral size and thickness of $\sim 500$ $\mathrm{nm}$ and $~ 5-6$ layers, respectively. We note that we do not achieve very high monolayer content. However, this is not a disadvantage: previous work has shown few-layer graphene to be a more effective reinforcing material than monolayer graphene [21]. To facilitate composite formation by melt processing, the exfoliated graphene was separated from the solvent to give a dry, weakly bound, reaggregated powder. Such powders are known to be very easy to re-exfoliate $[22,23]$.

The properties of polymer nanocomposites (Figure 1C) are controlled by a series of factors among which loading level, polymer-filler interfacial interactions, degree of exfoliation, dispersion and orientation of the nanofiller are key aspects [24, 25]. To assess both the exfoliation state and orientation of the exfoliated graphene layers within the PET matrix, TEM characterization was performed (Figure 1D-G). Unlike the situation shown in Figure 1A-B where the flakes observed were deposited from solution onto the TEM grid, in this case the samples analyzed were very thin sections $(\sim 100 \mathrm{~nm}$ thick) cut from the composite such that the orientation of the flakes within the composite was maintained. This allows both in-plane and out-of-plane orientations to be observed (Figure 1D-G). An extreme case is illustrated in Figure 1D where the flakes were orientated perpendicular to the cutting plane. Figure $1 \mathrm{G}$ displays isolated folded graphene layers encountered at a graphene content of $0.1 \mathrm{wt} \%$. This is unsurprising as folding of the graphene layers has been observed in the liquid phase (Figure 1A-B). However, the TEM images revealed little information about the 
dispersion of the graphene layers into the polymer matrix (Figure S1, Supporting Information), mostly due to the use of a low volume fraction of nanofiller.

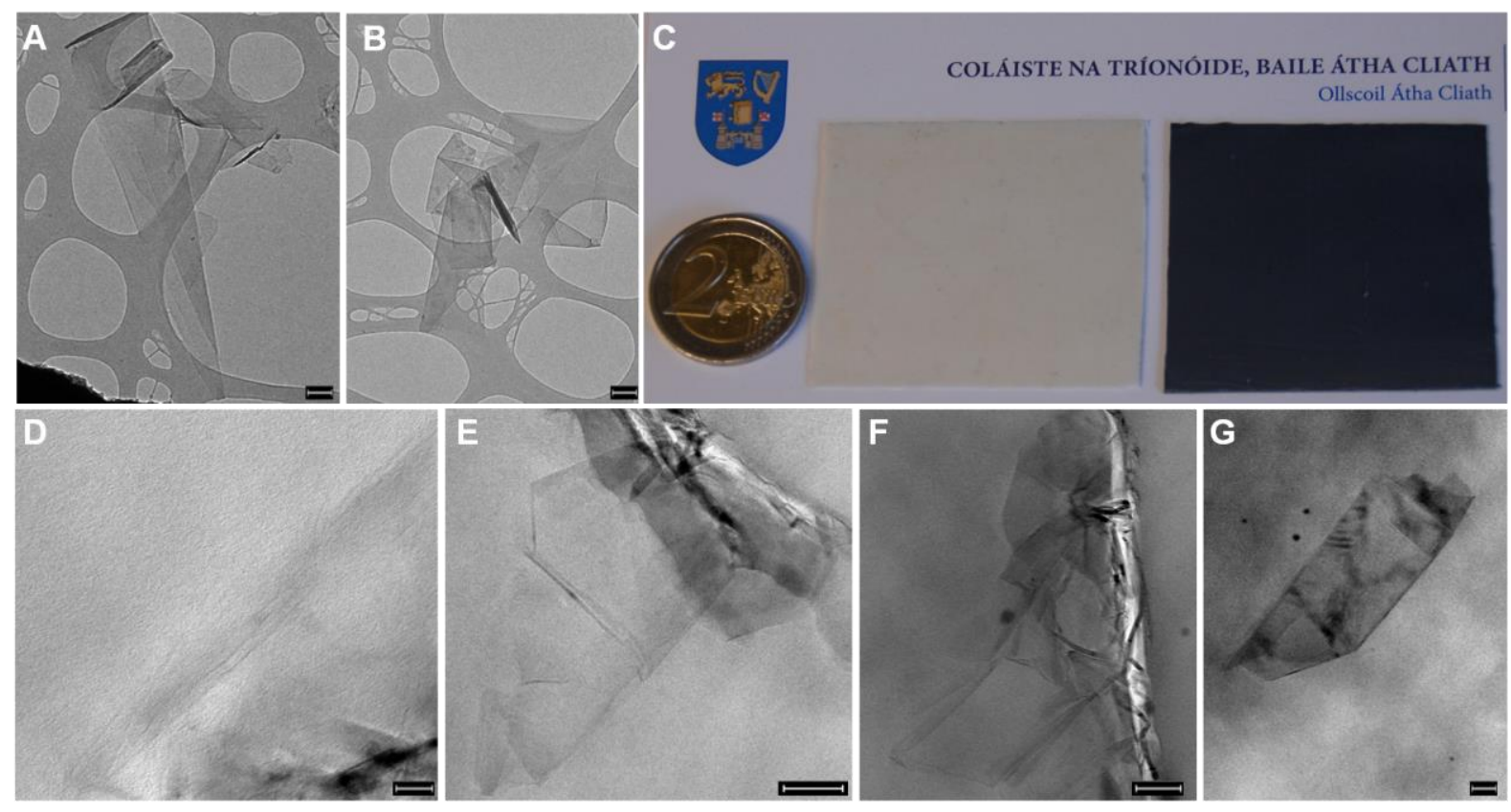

Figure 1. A-B) TEM images of exfoliated graphene layers. C) Left: PET and Right: PET/graphene nanocomposite manufactured via melt mixing with a graphene content of 0.07 wt\%; D-G) TEM images of graphene nanosheets embedded in a PET matrix. D) Graphene sheet orientated parallel to the beam $(0.07 \mathrm{wt} \%)$. E) Graphene sheet orientated perpendicular to the beam (0.07 wt\%). F) Graphene multilayer demonstrating layer delamination $(0.1 \mathrm{wt} \%)$. G) Folded graphene layers $(0.1 \mathrm{wt} \%)$. In all cases scale bars are $100 \mathrm{~nm}$.

The degree of dispersion of graphene flakes in the polymer matrix can be explored through microscopic examination of fracture surfaces. Shown in Figure 2 are scanning electron microscopy (SEM) and helium ion microscopy (HeIM) images of the surfaces exposed by tensile fracture. Both SEM and HeIM images exhibit a number of graphene flakes protruding from the matrix (Figure 2A-C). These flakes are well-isolated from each other illustrating the graphene flakes to be well dispersed within the PET matrix. We note that the presence of flakes protruding from fracture surfaces suggests the flakes to pullout from the matrix on failure. This implies the flakes to be shorter than the critical length of the reinforcement, defined as a multiple of the distance it takes for the strain to increase from the nanofiller's edges to the plateau region, and which was showed to be $\sim 3 \mu \mathrm{m}$ by Gong et al.[26, 27]. This is in line with previous work [26, 28]. 


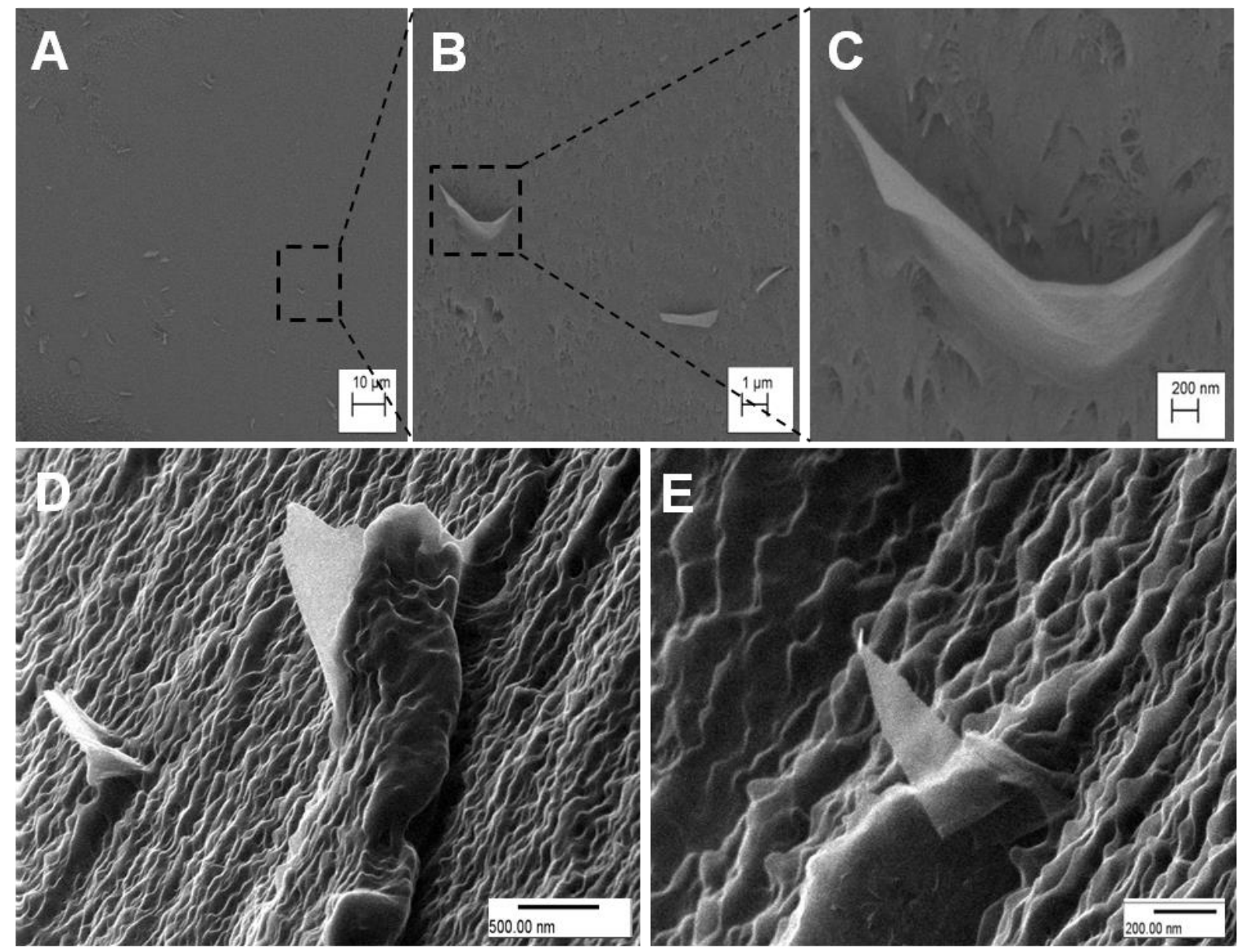

Figure 2. A-C) SEM images showing dispersion and delamination phenomena observed for $0.10 \mathrm{wt} \%$ PET/graphene nanocomposites. D-E) Helium ion micrographs of graphene sheets protruding from the fracture surface of PET reinforced with $0.10 \mathrm{wt} \%$ graphene.

The fact that the graphene flakes remain well-exfoliated and highly dispersed within the polymer matrix suggests these composites to potentially display mechanical reinforcement. The mechanical properties of PET and PET/graphene nanocomposites were measured by tensile testing and are summarized in Figure 3. The measured modulus increased slightly from $808 \mathrm{MPa}$ for neat PET to a maximum of $993 \mathrm{MPa}$ for the $0.08 \mathrm{wt} \%$ sample, an increase of $23 \%$ (Figure 3A). It is known that graphene displays an in-plane modulus of $1 \mathrm{TPa}$ [4]. Thus, assuming that the composite is filled with graphene flakes which are aligned in plane and are long enough for stress to transfer effectively from the polymer matrix to the graphene layers, the rule of mixtures would predict a modulus increase of $0.5 \mathrm{GPa}$ for $\mathrm{V}_{\mathrm{f}}=0.05 \%$ [29]. This is approximately 3 times larger than what was observed in the current study. However, this difference can be explained by a combination of random flake orientation and flake lengths which are too small to give complete stress transfer. 

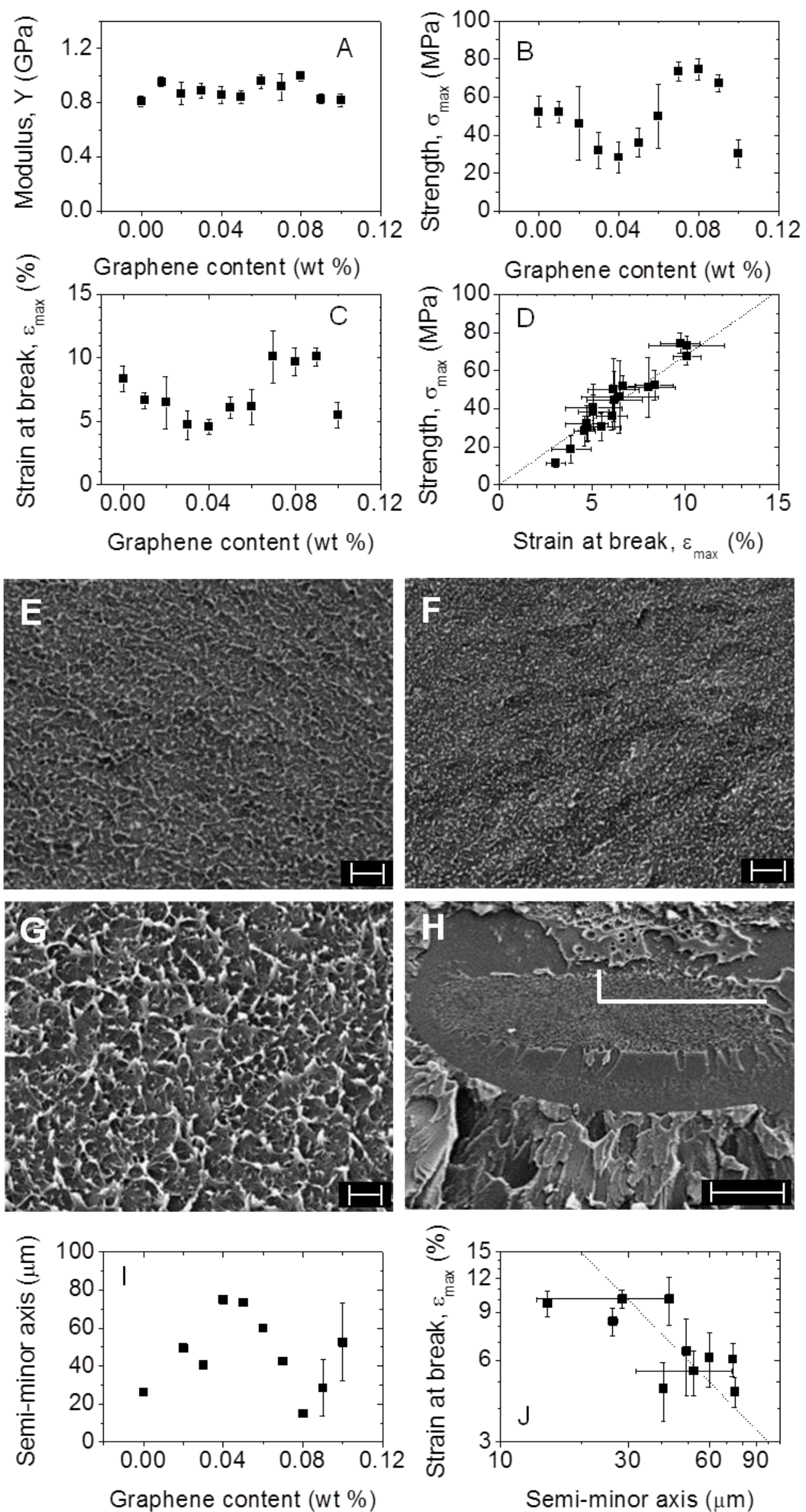
Figure 3. Mechanical and mechanistic data for neat PET and PET/graphene nanocomposites: A-C) Mechanical properties of PET and PET/graphene nanocomposites with a graphene layers concentration between 0.01 and $0.1 \mathrm{wt} \%$ : A) Tensile modulus; B) Tensile strength; C) Strain at break and D) Variation in the ultimate tensile strength with the strain at break; E-G) SEM images of the initiation area of the fracture surfaces of: E) PET; F) PET/graphene nanocomposite $(0.03 \mathrm{wt} \%)$ and G) PET/graphene nanocomposite (0.07 wt\%) (Scale bars: 1 $\mu \mathrm{m})$; H) Example of initiation area of the tensile fracture surface of PET/graphene nanocomposite $(0.02 \mathrm{wt} \%)$, the white lines represent the semi-minor and the semi-major axes of the ellipse shaped initiation area (Scale bar: $100 \mu \mathrm{m}$ ); I-J) Mechanistic data: I) Variation of the semi-minor axis of the initiation area with the graphene loading and J) Variation of the strain at break with the semi-minor axis of the initiation area.

The ultimate tensile strength displayed reproducibly complex behavior, first falling, then rising before falling again as the graphene content was increased (Figure 3B). However, it showed a net increase from $52 \mathrm{MPa}$ for PET to a maximum of $74 \mathrm{MPa}$ for the $0.08 \mathrm{wt} \%$ sample. This increase of $42 \%$ is much higher than that observed for the modulus although the maximum value occurs at the same graphene content. While this strength enhancement is lower than previous observations of a 2-3 fold enhancement of both modulus and strength for PET loaded with $\sim 0.5 \%$ graphene and functionalized graphene oxide [9, 15], it also occurs at a much lower loading level. To our knowledge no report has ever shown appreciable improvements in the mechanical properties of thermoplastic polymers at such low loadings as those seen here.

Interestingly, the strain at break displayed complex behavior similar to the strength (Figure 2C). Overall, a net increase from 8.4\% for PET to 10.1\% for PET reinforced with 0.07 wt\% graphene was observed. Typically, the dispersion of a nanofiller into a polymer matrix leads to a reduction in the elongation at break. While we observe this decrease at low volume fractions, as the graphene content was increased above $0.04 \mathrm{wt} \%$ the strain at break improved significantly.

The dispersion of only $0.07 \mathrm{wt} \%$ exfoliated graphene nanosheets in PET led to materials with better mechanical properties than previous melt-processed PET/graphene nanocomposites [30, 31]. In addition, the properties were equivalent to those found for nanocomposites prepared via solution processing of functionalized graphene oxide at much higher loadings ( 2 wt\%, Table S1, Supporting Information) [9]. Also, the extremely low volume fraction PET/graphene nanocomposites reported here exhibited mechanical properties 
similar or superior to those displayed by melt-mixed composites filled with other fillers or nanofillers such as organoclay, carbon nanotues, $\mathrm{TiO}_{2}$ or $\mathrm{SiO}_{2}$ that were directly melt mixed with PET (Table S2-S5, Supporting Information) [32-37]. However, we note that such performance is only found at low loading levels: increasing the graphene load above $0.1 \mathrm{wt} \%$ leads to a reduction in the mechanical properties, probably due to aggregation (Figure S3, Supporting Information). The depreciation of mechanical properties of polymer/graphene nanocomposites after a certain loading level has been previously reported in literature and attributed to the restacking of the graphene layers [38, 39].

The improvements in the mechanical properties of PET/graphene nanocomposites could possibly be attributed to the superior mechanical properties displayed by the graphene sheets or their high surface area [2, 4, 16, 40-44]. To differentiate between these effects, PET with $0.07 \mathrm{wt} \%$ graphite was melt blended in the same conditions (Figure S4, Supporting Information). The results showed that the ultimate tensile strength decreased by approximately 50\%. This shows the importance of filler thickness, which in our case is related to the exfoliation state, and it also implies that filler surface area may be important.

The fact that both strength and strain at break display the same type of complex behavior (Figure 3D) suggests the presence of graphene to influence the fracture process, even at very low loading levels. In the neat polymer, fracture is known to be preceded by fibrillation and stress induced crystallization [45, 46]. The fracture makes the fibrils visible and measurement of the characteristics of these fibrils can shed light on the influence of graphene on the fracture process. Using SEM to analyze the area where the tensile fracture initiated (Figure 3E-G) it can be observed that fibrils are present. Fibrillation indicates slips within the crystalline lamellae and changes in the chain direction [47]. The occurrence of fibrils may suggest that the break was due to crazing either in the amorphous area (short fibrils) or in the semi-crystalline area (long fibrils) [48]. While, the height of the fibrils cannot be directly measured, their appearance, as observed via SEM, may give some indication of the breaking mechanism. For neat PET, the fibrils appear to be relatively fine (Figure 3E), implying fracture to be associated with the amorphous region. At extremely low graphene content $(0.03 \mathrm{wt} \%)$, the fibrils are even finer with a representative image shown in Figure 3F. At such low volume fractions, isolated graphene nanosheets may hinder the reorientation of the polymer chains, thus splitting the material into molecular bundles and cavities and leading to local failure and ultimately premature fracture. Thus, upon imaging the specimen a finer fibrillation may be observed. The finer fibrillation is characteristic of a more brittle aspect, as previously reported in literature for polymer/clay nanocomposites [49]. However, increasing 
the amount of graphene to $0.07 \mathrm{wt} \%$, had a significant effect on the local structure in the initiation area with considerably larger fibrils observed (Figure 3G). The increased height of the fibrils suggests that, in this case, the fracture may be associated with the semi-crystalline area. We hypothesize that the presence of graphene acts to reinforce the stress-induced crystallites leading them to fail later than would otherwise be the case. This has been previously reported in the literature and attributed to the presence of graphene which inhibits the strain induced crystallization $[9,50]$. This is consistent with the observed increase in strain at break (Figure 3C).

This clearly shows the presence of graphene to significantly affect the microstructure of the adjacent polymer as seen clearly by changes to the aspect of the fibrils. While increased fibrillation with improved tensile strength has been previously reported in literature for PET/clay nanocomposites, their appearance has not been quantified [51]. However, Yuan and Misra reported that the presence of clay in a semi-crystalline polymer matrix led to insignificant changes in the crystallinity, significant reduction of sperulite sizes, improved yield strength and enhanced impact toughness [52]. Their analysis on the impact fractured surfaces reveals that polymer/clay nanocomposites displayed a smaller fracture initiation zone and enhanced fibrillation in the crack propagation zone, compared to the neat polymer. Although we cannot directly measure the height of the fibrils at the center of the nucleus, we can perform detailed characterization on the properties of the initiation area. We expect an inverse correlation between fibril height and spatial dimensions of the initiation area because the size of the initiation area tends to propagate as fibrils break. Thus, early breakage leads to faster propagation and so larger initiation area. As can be observed from Figure $3 \mathrm{H}$, the initiation area can be approximated as an ellipse, and characterized by a semi-minor and a semi-major axis (represented by white lines). As the height of the fibrils appeared to change with the addition of graphene nanolayers in PET (Figure 3F-G vs. Figure 3E), the dimensions of the semi-axes (Figure 3H) of the ellipses also varied (Figure 3I and Figure S6, Supporting Information). The neat polymer presented a semi-minor axis of $26 \mu \mathrm{m}$. The addition of 0.04 wt\% graphene layers enhanced the semi-minor axis to $75 \mu \mathrm{m}$. This is consistent with the decrease observed in the height of the fibrils (Figure 3F vs. Figure 3E), and suggests that the reorientation of the polymer chains was obstructed by the presence of the graphene nanolayers as described above. This led to small fibrils that were quickly severed under strain leading to rapid growth of the initiation area. As the graphene content was increased above $0.04 \mathrm{wt} \%$ and the stress and strain-at-break began to increase (Figure 3B-C), the sample presented pronounced fibrillation (Figure 3G) and the semi-minor axis decreased to $15 \mu \mathrm{m}$ for PET with 
$0.08 \mathrm{wt} \%$ exfoliated graphene layers. This suggested that the stress was transferred from the polymer to the graphene layers, which delaminated creating more surfaces and allowing the material to withstand higher loads. The delamination process subsequently prohibited the development of the nucleus and resulted in a smaller initiation area.

To properly establish the relationship between the mechanical properties and the fracture surface, the strain at break was plotted as a function of the semi-axes of the initiation area (Figure 3J and Figure S7, Supporting Information). As can be observed from Figure 3J there is an anti-correlation between the semi-minor axis and the strain at break. At optimal exfoliated graphene contents (i.e., $0.08 \mathrm{wt} \%$ ), delamination of the graphene layers prevents the enhancement of the initiation area whilst withstanding higher loads. However, at low graphene layers contents (i.e., $0.04 \mathrm{wt} \%$ ) the initiation area quickly propagates, the presence of graphene prohibiting the reorientation of polymer chains and leading to a premature break of the polymer chains.

\section{Conclusions}

In summary, polymer/graphene nanocomposites with superior mechanical properties were manufactured via melt processing using an extremely low loading level of exfoliated graphene layers (i.e., less than $0.1 \mathrm{wt} \%$ ) and by carefully choosing a polymer matrix. We find that for this semi-crystalline polymer matrix and liquid exfoliated graphene there is a direct correlation between the tensile strength and the strain at break and an indirect correlation between the tensile strength and the dimensions of the initiation area, and subsequently the degree of fibrillation in the area. This approach may lead to the industrial production of polymer/graphene nanocomposites with minimal nanofiller contents and desirable mechanical properties.

\section{Appendix A. Supplementary data}

Supplementary data is available.

\section{Acknowledgements}

The authors acknowledge the financial support from Science Foundation Ireland, through the CRANN Pathfinder program. The Conway Institute is thanked for aiding with the sample cryo-ultramicrotoming and facilitating access to the TEM.

\section{References}


[1] Geim AK, Novoselov KS. The rise of graphene. Nat Mater. 2007;6(3):183-91.

[2] Zhu Y, Murali S, Cai W, Li X, Suk JW, Potts JR, Ruoff RS. Graphene and Graphene Oxide: Synthesis, Properties, and Applications. Adv Mater. 2010;22(35):3906-24.

[3] Frank O, Bouša M, Riaz I, Jalil R, Novoselov KS, Tsoukleri G, Parthenios J, Kavan L, Papagelis K, Galiotis C. Phonon and Structural Changes in Deformed Bernal Stacked Bilayer Graphene. Nano Lett. 2011;12(2):687-93.

[4] Lee C, Wei X, Kysar JW, Hone J. Measurement of the Elastic Properties and Intrinsic Strength of Monolayer Graphene. Science. 2008;321(5887):385-8.

[5] Kuilla T, Bhadra S, Yao DH, Kim NH, Bose S, Lee JH. Recent advances in graphene based polymer composites. Prog Polym Sci. 2010;35(11):1350-75.

[6] Li M, Jeong YG. Poly(ethylene terephthalate)/exfoliated graphite nanocomposites with improved thermal stability, mechanical and electrical properties. Composites Part A: Applied Science and Manufacturing. 2011;42(5):560-6.

[7] Potts JR, Dreyer DR, Bielawski CW, Ruoff RS. Graphene-based polymer nanocomposites. Polymer. 2011;52(1):5-25.

[8] Du J, Cheng H-M. The Fabrication, Properties, and Uses of Graphene/Polymer Composites. Macromolecular Chemistry and Physics. 2012;213(10-11):1060-77.

[9] Shim SH, Kim KT, Lee JU, Jo WH. Facile Method to Functionalize Graphene Oxide and Its Application to Poly(ethylene terephthalate)/Graphene Composite. ACS Applied Materials \& Interfaces. 2012;4(8):4184-91.

[10] Kim H, Kobayashi S, AbdurRahim MA, Zhang MJ, Khusainova A, Hillmyer MA, Abdala AA, Macosko CW. Graphene/polyethylene nanocomposites: Effect of polyethylene functionalization and blending methods. Polymer. 2011;52(8):1837-46.

[11] El Achaby M, Arrakhiz F-E, Vaudreuil S, el Kacem Qaiss A, Bousmina M, FassiFehri O. Mechanical, thermal, and rheological properties of graphene-based polypropylene nanocomposites prepared by melt mixing. Polymer Composites. 2012;33(5):733-44.

[12] Vallés C, Kinloch IA, Young RJ, Wilson NR, Rourke JP. Graphene oxide and basewashed graphene oxide as reinforcements in PMMA nanocomposites. Compos Sci Technol. 2013;88(0):158-64.

[13] El Achaby M, Qaiss A. Processing and properties of polyethylene reinforced by graphene nanosheets and carbon nanotubes. Materials \& Design. 2013;44(0):81-9.

[14] Jin J, Rafiq R, Gill YQ, Song M. Preparation and characterization of high performance of graphene/nylon nanocomposites. European Polymer Journal. 2013;49(9):2617-26. 
[15] Khan U, Young K, O'Neill A, Coleman JN. High strength composite fibres from polyester filled with nanotubes and graphene. Journal of Materials Chemistry. 2012;22(25):12907-14.

[16] Terrones M, Martín O, González M, Pozuelo J, Serrano B, Cabanelas JC, Vega-Díaz SM, Baselga J. Interphases in Graphene Polymer-based Nanocomposites: Achievements and Challenges. Adv Mater. 2011;23(44):5302-10.

[17] Hernandez Y, Nicolosi V, Lotya M, Blighe FM, Sun Z, De S, McGovern IT, Holland B, Byrne M, Gun'Ko YK, Boland JJ, Niraj P, Duesberg G, Krishnamurthy S, Goodhue R, Hutchison J, Scardaci V, Ferrari AC, Coleman JN. High-yield production of graphene by liquid-phase exfoliation of graphite. Nat Nano. 2008;3(9):563-8.

[18] Nicolosi V, Chhowalla M, Kanatzidis MG, Strano MS, Coleman JN. Liquid Exfoliation of Layered Materials. Science. 2013;340(6139):1420-+.

[19] Paton KR, Varrla E, Backes C, Smith RJ, Khan U, O’Neill A, Boland C, Lotya M, Istrate OM, King P, Higgins T, Barwich S, May P, Puczkarski P, Ahmed I, Moebius M, Pettersson H, Long E, Coelho J, O'Brien SE, McGuire EK, Mendoza-Sanchez B, Duesberg GS, McEvoy N, Pennycook TJ, Downing C, Crossley A, Nicolosi V, Coleman JN. Scalable production of large quantities of defect-free, few-layer graphene by shear exfoliation in liquids Nat Mater. 2014.

[20] Khan U, O'Neill A, Lotya M, De S, Coleman JN. High-Concentration Solvent Exfoliation of Graphene. Small. 2010;6(7):864-71.

[21] Gong L, Young RJ, Kinloch IA, Riaz I, Jalil R, Novoselov KS. Optimizing the Reinforcement of Polymer-Based Nanocomposites by Graphene. ACS Nano. 2012;6(3):208695.

[22] Barwich S, Khan U, Coleman JN. A Technique To Pretreat Graphite Which Allows the Rapid Dispersion of Defect-Free Graphene in Solvents at High Concentration. The Journal of Physical Chemistry C. 2013;117(37):19212-8.

[23] Khan U, Porwal H, O'Neill A, Nawaz K, May P, Coleman JN. Solvent-Exfoliated Graphene at Extremely High Concentration. Langmuir. 2011;27(15):9077-82.

[24] Park S-J, Seo M-K. Chapter 8 - Composite Characterization. In: Soo-Jin P, Min-Kang S, eds. Interface Sci Technol: Elsevier 2011, p. 631-738.

[25] Pividori MI, Alegret S. Chapter 4- Gold Nanocomposites. In: Kumar CSSR, ed. Nanocomposites. Darmstadt, Germany: Wiley+VCH Verlag GmbH\&Co. KGaA 2010, p. 13968. 
[26] Gong L, Kinloch IA, Young RJ, Riaz I, Jalil R, Novoselov KS. Interfacial Stress Transfer in a Graphene Monolayer Nanocomposite. Adv Mater. 2010;22(24):2694-7.

[27] Kelly A, Macmillan NH. Strong Solids. 3rd ed. Oxford: Clarendon Press; 1986.

[28] May P, Khan U, O'Neill A, Coleman JN. Approaching the theoretical limit for reinforcing polymers with graphene. Journal of Materials Chemistry. 2012;22(4):1278-82.

[29] Padawer GE, Beecher N. On Strength and Stiffness of Planar Reinforced Plastic Resins. Polymer Engineering and Science. 1970;10(3):185-\&.

[30] Bandla S, Hanan J. Microstructure and elastic tensile behavior of polyethylene terephthalate-exfoliated graphene nanocomposites. J Mater Sci. 2012;47(2):876-82.

[31] Al-Jabareen A, Al-Bustami H, Harel H, Marom G. Improving the oxygen barrier properties of polyethylene terephthalate by graphite nanoplatelets. Journal of Applied Polymer Science. 2013;128(3):1534-9.

[32] Wang Y, Gao J, Ma Y, Agarwal US. Study on mechanical properties, thermal stability and crystallization behavior of PET/MMT nanocomposites. Composites Part B: Engineering. 2006;37(6):399-407.

[33] Todorov LV, Viana JC. Characterization of PET nanocomposites produced by different melt-based production methods. Journal of Applied Polymer Science. 2007;106(3):1659-69.

[34] Chen L, Liu K, Jin TX, Chen F, Fu Q. Rod like attapulgite/poly(ethylene terephthalate) nanocomposites with chemical bonding between the polymer chain and the filler. Express Polymer Letters. 2012;6(8):629-38.

[35] Nanni F, Mayoral BL, Madau F, Montesperelli G, McNally T. Effect of MWCNT alignment on mechanical and self-monitoring properties of extruded PET-MWCNT nanocomposites. Compos Sci Technol. 2012;72(10):1140-6.

[36] Abu-Isa IA, Jaynes CB, Ogara JF. High-impact-strength poly(ethylene terephthalate) (PET) from virgin and recycled resins. Journal of Applied Polymer Science. 1996;59(13):1957-71.

[37] Li N, Luo P, Liu K, Chen L, Wang K, Chen F, Fu Q. Preparation and properties of poly(ethylene terephthalate)/inorganic whiskers composites. Journal of Applied Polymer Science. 2011;121(1):604-11.

[38] Zhao X, Zhang Q, Chen D, Lu P. Enhanced Mechanical Properties of Graphene-Based Poly(vinyl alcohol) Composites. Macromolecules. 2010;43(5):2357-63. 
[39] Song P, Cao Z, Cai Y, Zhao L, Fang Z, Fu S. Fabrication of exfoliated graphene-based polypropylene nanocomposites with enhanced mechanical and thermal properties. Polymer. 2011;52(18):4001-10.

[40] Bao Q, Zhang H, Yang J-X, Wang S, Tang DY, Jose R, Ramakrishna S, Lim CT, Loh KP. Graphene-Polymer Nanofiber Membrane for Ultrafast Photonics. Adv Funct Mater. 2010;20(5):782-91.

[41] Martin-Gallego M, Bernal MM, Hernandez M, Verdejo R, Lopez-Manchado MA. Comparison of filler percolation and mechanical properties in graphene and carbon nanotubes filled epoxy nanocomposites. Eur Polym J 2013;49(6):1347-53.

[42] Rafiee MA, Rafiee J, Wang Z, Song H, Yu Z-Z, Koratkar N. Enhanced Mechanical Properties of Nanocomposites at Low Graphene Content. ACS Nano. 2009;3(12):3884-90. [43] Yang X, Li L, Shang S, Tao X-m. Synthesis and characterization of layer-aligned poly(vinyl alcohol)/graphene nanocomposites. Polymer. 2010;51(15):3431-5.

[44] Yang X, Tu Y, Li L, Shang S, Tao X-m. Well-Dispersed Chitosan/Graphene Oxide Nanocomposites. ACS Applied Materials \& Interfaces. 2010;2(6):1707-13.

[45] Misra A, Stein RS. Stress-induced crystallization of poly(ethylene terephthalate). Journal of Polymer Science: Polymer Physics Edition. 1979;17(2):235-57.

[46] Ronkay F, Czigány T. Cavity formation and stress-oscillation during the tensile test of injection molded specimens made of PET. Polym Bull. 2006;57(6):989-98.

[47] Hiss R, Hobeika S, Lynn C, Strobl G. Network Stretching, Slip Processes, and Fragmentation of Crystallites during Uniaxial Drawing of Polyethylene and Related Copolymers. A Comparative Study. Macromolecules. 1999;32(13):4390-403.

[48] Deblieck RAC, van Beek DJM, Remerie K, Ward IM. Failure mechanisms in polyolefines: The role of crazing, shear yielding and the entanglement network. Polymer. 2011;52(14):2979-90.

[49] Nathani H, Dasari A, Misra RDK. On the reduced susceptibility to stress whitening behavior of melt intercalated polybutene-clay nanocomposites during tensile straining. Acta Mater. 2004;52(11):3217-27.

[50] Taniguchi A, Cakmak M. The suppression of strain induced crystallization in PET through sub micron TiO2 particle incorporation. Polymer. 2004;45(19):6647-54.

[51] Hwang SY, Lee WD, Lim JS, Park KH, Im SS. Dispersibility of clay and crystallization kinetics for in situ polymerized PET/pristine and modified montmorillonite nanocomposites. Journal of Polymer Science Part B: Polymer Physics. 2008;46(11):1022-35. 
[52] Yuan Q, Misra RDK. Impact fracture behavior of clay-reinforced polypropylene nanocomposites. Polymer. 2006;47(12):4421-33. 